\title{
Equivalent of chromium
}

\section{Lefort}

To cite this article: M. Lefort (1850) Equivalent of chromium, Philosophical Magazine Series 3, 36:244, 407-407, DOI: 10.1080/14786445008646512

To link to this article: http://dx.doi.org/10.1080/14786445008646512

曲 Published online: 30 Apr 2009.

Submit your article to this journal

Џ Article views: 3

Q View related articles $₫$ 
tion is very distinct. This compound is white; on treating it with potash, there are produced ferrocyanide of potassium and yellow oxide of mercury, insoluble in excess of potash.-L'Institut, Avril 10, $1850^{\circ}$.

\section{EQUIVALENT OF CHROMIUM. BY M. LEFORT.}

According to Berzelius, the equivalent of chromium is 351 ; other chemists make it 320 and 330 : according to $M$. Lefort, the equivalent is $333 .-$ Ibid.

\section{METEOROLOGICAL OBSERVATIONS FOR MARCH 1850.}

Chiswick.-March 1. Hazy : very fine: overcast. 2. Densely overcast. 3. Cloudy. 4. Cloudy : clear and frosty at night. 5. Frosty : fine: cloudy. 6, 7. Foggy : fine: clear. 8. Hazy. 9. Slight fog : fine : clear. 10. Very fine. 11. Clear and fine: frosty. 12. Frosty : very fine: clear. 13. Frosty, with slight fog : very fine. 14. Overcast. 15. Overcast : clear, with sharp frost at night. 16. Frosty : overcast : clear. 17. Frosty : cloudy : sunshine occasionally : clear and frosty. 18. Frosty and fine. 19,20. Cloudy. 21. Cloudy and cold : clear and frosty. 22. Hoar-frost : cloudy. 23. Heavy clouds : sleet-showers. 24. Slight fall of snow-flakes : severe frost at night. 25. Clear and frosty : cloudy : clear : frosty. 26. Overcast. 27. Foggy : slight haze : frosty. 28. Slight laze : bright sun with dry cold air: clear and frosty. 29. Foggy: cloudy : clear. 30, 31 . Overcast.

Mean temperature of the month $\ldots . \ldots \ldots \ldots \ldots \ldots \ldots \ldots \ldots . . . \ldots . \ldots 7^{\circ} \cdot 71$

Mean temperature of March 1849 ............................. $41 \cdot 56$

Mean temperature of March for the last twenty-three years $42 \cdot 81$

Average amount of rain in March ........................... 1.36 inch.

Boston. - March 1,2. Cloudy. 3. Fine: rain P.M. 4. Cloudy. 5-7. Fine. 8, 9. Clondy, 10. Fine. 11. Cloudy. 12, 13. Fine. 14, 15. Cloudy. 16. Cloudy : rain A.ss. 17, 18. Fine. 19. Cloudy : rain A.s. 20. Cloudy. 21. Fine. 22. Cloudy. 23. Fine: rain early A.M. 24. Cloudy: snow A.M. and s. M. 25. Cloudy, 26, 27. Fine. 28. Fine: rain A.M. 29, 30. Fine. 31. Cloudy : rain $A . M$.

Applegarth Manse, Dumfries-shire.-March 1. Fine: a shower early A.M. 2. Slight shower during night: thick P.M. 3. Rain heavy A.M. : cleared. 4. Frost : clear and fine. 5. Moist all day. 6. Mild and growing: moist. 7. Fine spring day. 8. Still finer: clear and warm. 9. Dull and moist : slight shower. 10 . Clear and fine : slight shower. 11. Frost : clear and bright. 12. Frost : cloudy. 13. Frost, not so severe. 14, 15. Mild : cluudy : no frost. 16. Eust wind : dull. 17. Dull : raw : cold : shower. 18. Dull and cloudy, but mild. 19. Dull A.M.: cleared : rain r.M. 20. Very fine : slight drizzle. 21. Fine, though raw A.M. 22. Dry, but cloudy : stormy P.M. 23. Frost: snow: wind. 24. Hard frost : snow : calm P.m. 25. Frost very hard : thermometer $211^{\circ}$. 25. Frost slight : heavy snow A.M. 27. Frost severe : snow again. 28. Frost still harder : clear. 29. Frost still. 30. Frost moderate : hail : rain P.M. 31. Rain : cloudy and foggy P.M.

Mean temperature of the month ............................ $40^{\circ} \cdot 3$

Mean temperature of March 1849 ........................... 41 : 8

Mean temperature of March for the last twenty-eight years. $39 \cdot 6$

Average rain in March for twenty -three years ............... 2.35 inches.

Sandwick Manse, Orkney.-March 1. Showers : drops. 2. Drizzle. 3. Cloudy : showers. 4. Snow : cloudy. 5. Damp : rain. 6. Drizzle : cloudy. 7. Damp. 8. Fine : drizzle. 9. Cloudy : showers. 10. Sleet : aurora. 11. Bright: clear : aurora. 12. Drizzle. 13. Damp : drizzle. 14. Drizzle: damp. 15. Bright : damp. 16. Cloudy, 17. Drops : showers. 18. Hazy : cloudy. 19. Cloudy. 20. Bright : drops. 2l. Cloudy : drops. 22. Showers : snow-showers. 29. Hail-showers: snow-showers. 24. Snow-drift: snow-show'ers. 25. Snowshowers : large halo. 26. Snow-showers. 27, 28. Bright: snow-showers. 29. Bright : clear. 30. Cloudy. 31. Dropss : cloudy. 